\title{
Prevalence of Hemoplasma Infection among Cattle in the Western Part of Japan
}

\author{
Yu FUJIHARA ${ }^{1)}$, Fumina SASAOKA $^{1)}$, Jin SUZUKI ${ }^{1)}$, Yusaku WATANABE ${ }^{1)}$, Masatoshi FUJIHARA ${ }^{1)}$, \\ Katsufumi OOSHITA ${ }^{2)}$, Hitoshi $\mathrm{ANO}^{3)}$ and Ryô HARASAWA ${ }^{1) *}$ \\ ${ }^{1)}$ Department of Veterinary Microbiology, Faculty of Agriculture, Iwate University, Morioka 020-8550, ${ }^{2)}$ Hatsukaichi Branch of \\ Yamagata Veterinary Clinical Center, NOSAI Hiroshima, Hatsukaichi, Hiroshima 738-0015 and ${ }^{3)}$ Department of Veterinary Internal \\ Medicine, Faculty of Agriculture, Miyazaki University, Miyazaki 889-2192, Japan
}

(Received 5 June 2011/Accepted 15 July 2011/Published online in J-STAGE 29 July 2011)

ABSTRACT. We have examined for hemoplasma infection among cattle in the Hiroshima and Miyazaki prefectures by using a sensitive real-time PCR, with SYBR Green I and with melting curve analysis, which allow to distinguish the two bovine hemoplasma species, Mycoplasma wenyonii and 'Candidatus M. haemobos'. We found $69.4 \%$ of 36 cattle in Hiroshima and $93.8 \%$ of 32 cattle in Miyazaki infected with either of these two hemoplasma species. High morbidity in western part of Japan may reflect the activity of arthropod vectors for hemoplasma transmission. We also demonstrated neonatal calves less than three months old affected with hemoplasmas without grazing in summer, suggesting a possibility of vertical transmission.

KEY WORDS: hemoplasma, mycoplasma, vertical transmission.

Only two hemoplasma species, Mycoplasma wenyonii and 'Candidatus M. haemobos' (synonym for ' $C$. M. haemobovis') are currently recognized in cattle $[6,9]$. Although $M$. wenyonii has been shown to exhibit worldwide geographical distribution, ' $C$. M. haemobos' has been solely reported from Switzerland, China, Germany and Japan $[2,3,8,9]$. Both hemoplasma species are causative of infectious anemia in cattle to some extent and have been demonstrated in Hokkaido and Tohoku regions of this country. Incidences of $M$. wenyonii and ' $C$. M. haemobos' infections have been reported as 21.8 and $16.7 \%$, respectively, in Hokkaido, and 40.3 and $56.8 \%$, respectively, in the Miyagi prefecture [7, 9]. Infection has spread by transmission routes that we do not fully understand. Despite the relatively high incidence in these areas, little is known about the prevalence of bovine hemoplasma infections in western parts of Japan. Therefore, we examined for hemoplasma prevalence among cattle in the Hiroshima and Miyazaki prefectures by using a sensitive real-time PCR to detect the 16S rRNA gene [4], with SYBR Green I and with melting curve analysis, which allow to distinguish these two hemoplasma species.

EDTA-anticoagulated or heparinized blood samples from 68 cattle in different herds of the Hiroshima and Miyazaki prefectures were randomly collected between 2008 and 2011. Information on clinical diagnoses and age of all the cattle included in this study was obtained from the relevant veterinarians or owners. The ages of cattle were ranging from one month to 15 years old. Cattle included less than yearling in both the prefectures. Total DNA was extracted from $200 \mu l$ whole blood samples by using the QIAamp

\footnotetext{
* Correspondence to: Harasawa, R., Department of Veterinary Microbiology, Faculty of Agriculture, Iwate University, Morioka, Iwate 020-8550, Japan. e-mail: harasawa-tky@umin.ac.jp

\# These authors (Y. F. and F. S.) contributed equally to this work.
}

DNA Blood Mini Kit (Qiagen, Hilden, Germany) according to the manufacturer's instructions. Negative controls consisting of $200 \mu l$ phosphate-buffered saline solution were included with each batch. Extracted DNA samples were stored at $-20^{\circ} \mathrm{C}$ prior to examination.

To detect the both hemoplasma species in real-time PCR, specific primers for the $16 \mathrm{~S}$ rRNA gene were used as described previously [7]. Forward primer, 5'-ATATTCCTACGGGAAGCAGC-3', equivalent to nucleotide numbers 328 to 347 of $M$. wenyonii and reverse primer, 5'ACCGCAGCTGCTGGCACATA-3', equivalent to nucleotide numbers 503 to 522 of $M$. wenyonii amplified a 195 and 173 bp for $M$. wenyonii and ' $C$. M. haemobos', respectively. Nucleotide sequences and sizes bracketed by the primers are peculiar to each hemoplasma. The 22-bp gap in the PCR product from ' $C$. M. haemobos' attributes to a genetic marker to distinguish it from $M$. wenyonii in the real-time PCR [7].

Real-time PCR was performed in a SmartCycler instrument (Cepheid, Sunnyvale, CA, U.S.A.) with SYBR Premix Ex Taq (Code \#RR041A, TaKaRa Bio., Shiga, Japan). The reaction mixture contained $1 \mu l$ of each primer $(10 \mathrm{pmol} / \mu l)$, $12.5 \mu \mathrm{l}$ of $2 \mathrm{X}$ premix reaction buffer and water to volume of $23 \mu l$. Finally, $2 \mu l$ of DNA samples as templates were added to this mixture. Amplification was achieved with 40 cycles of denaturation at $95^{\circ} \mathrm{C}$ for $5 \mathrm{sec}$, renaturation at $57^{\circ} \mathrm{C}$ for $20 \mathrm{sec}$, and elongation at $72^{\circ} \mathrm{C}$ for $15 \mathrm{sec}$, after the initial denaturation at $94^{\circ} \mathrm{C}$ for $30 \mathrm{sec}$. Positive cattle were found affected with each hemoplasma irrespective of the age.

After real-time PCR, melting experiment was performed from 60 to $95^{\circ} \mathrm{C}$ at $0.2^{\circ} \mathrm{C} / \mathrm{sec}$ with smooth curve setting averaging one point. Melting peaks were visualized by plotting the first derivative against the melting temperature $(\mathrm{Tm})$ as described previously [1]. Melting curve analysis of the amplified products allowed differentiation of these two 
hemoplasma species, since nucleotide sequences and sizes bracketed by the primers are distinct between the species. Thus, the variations in the $T m$ may serve as a differential marker for hemoplasma species. The input amount of DNA, the copy number of the target as well as presence of co-infections with several targets did not influence the Tm. No melting peak was evident on negative cattle.

In the present study, we found overall $69.4 \%(25 / 36)$ and $93.8 \%(30 / 32)$ cattle infected with hemoplasmas in Hiroshima and Miyazaki, respectively (Fig. 1). This incidence was much higher than that in Hokkaido and Miyagi, suggesting factors in geographical latitude affected in infections. The geographical difference in morbidity may attribute to activity of arthropod vectors for hemoplasma transmission. ' $C$. M. haemobos' infection was predominant in both the prefectures. Thirty-six cattle in Hiroshima included 18 stores from yearling to two years old and 18 adult dairy cows. Despite missing exact age, no relationship to infections was evident among age and bleed of cattle in Hiroshima. In Miyazaki, although extremely high incidence of infections was demonstrated over all, relatively low incidence was found in elder cattle. More surprising result is that all the calves less than three months born in winter were unexpectedly infected with ' $C$. M. haemobos' (Table 1). It raises a question of whether vectors for the hemoplasma transmission are unnecessary, since ticks have been suspected as a vector for feline hemoplasma infection [10]. Given the absence of vectors in winter, it will turn out that vertical transmission is most likely route of neonatal infection, since cattle may become infection while grazing in summer.

The hemoplasma-infected cattle in the present study did not exhibit clinical signs such as anemia attributable to hemoplasmosis, though hemoplasma infections in cattle were first recognized in Swiss dairy cows with hemolytic anemia [3]. In our study, no significant association was found between the infection status and anemic syndromes. One possibility is that there might be distinct strains in virulence or anemia might be caused by opportunistic infections. As none of the PCR-positive cattle caused clinical manifestations of severe anemia or all cattle had been presented for reasons unrelated to hemoplasmosis, laboratory parameters were not subjected to in-depth investigation. Although our results indicate wide distribution of $M$. wenyonii and ' $C$. M. haemobos' among cattle population in this country without developing anemic syndromes, infected animals probably remain chronic carriers after clinical signs have resolved. Thus, the persistent infections with hemoplasmas may contribute to the progression of retroviral, neoplastic, or immune-mediated diseases [5]. Collectively, our findings suggest possibility of vertical transmission because of neonatal infections and monitoring of hemoplasma infection may help to maintain hygienic conditions for cattle production.

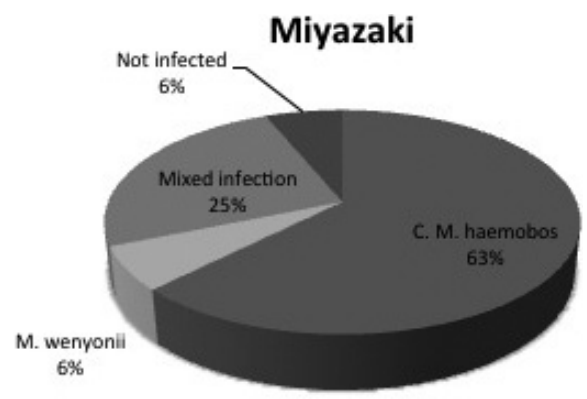

Fig. 1. Hemoplasma infections among cattle in Hiroshima and Miyazaki prefectures. Percentages were shown in round figures.

Table 1. Incidence of hemoplasma infections in cattle of the Miyazaki Prefecturre

\begin{tabular}{lccc}
\hline Age & No. of cattle examined & M. wenyonii & 'C. M. haemobos' \\
\hline Less than 3 months & 4 & 0 & 4 \\
Yearling & $23^{* *}$ & $7^{*}$ & $21^{*}$ \\
2 years old & 1 & 0 & 1 \\
7 years old & 1 & 0 & 0 \\
11 years old & 1 & $1^{*}$ & $1^{*}$ \\
14 years old & 1 & 1 & 0 \\
15 years old & 1 & $1^{*}$ & $1^{*}$ \\
\hline Total & 32 & $10(31.3 \%)$ & $28(87.5 \%)$ \\
\hline
\end{tabular}

* Numbers include mixed infections. ** One of the yearlings was not infected with either of the hemoplasma species. 


\section{REFERENCES}

1. Harasawa, R., Mizusawa, H., Fujii, M., Yamamoto, J., Mukai, H., Uemori, T., Asada, K. and Kato, I. 2005. Rapid detection and differentiation of the major mycoplasma contaminants in cell cultures using real-time PCR with SYBR Green I and melting curve analysis. Microbiol. Immunol. 49: 859-863.

2. Hoelzle, K., Winkler, M., Kramer, M. M., Wittenbrink, M. M., Dieckmann, S. M. and Hoelzle, L. E. 2011. Detection of Candidatus Mycoplasma haemobos in cattle with anaemia. Vet. J. 187: 408-410.

3. Hofmann-Lehmann, R., Meli, M. L., Dreher, U. M., Gönczi, E., Deplazes, P., Braun, U., Engels, M., Schüpbach, J., Jörger, K., Thoma, R., Griot, C., Stark, K. D. C., Willi, B., Schmidt, J., Kocan, K. M. and Lutz, H. 2004. Concurrent infections with vector-borne pathogens associated with fetal hemolytic anemia in a cattle herd in Switerland. J. Clin. Microbiol. 42: 37753780 .

4. McAuliffe, L., Lawes, J., Bell, S., Barlow, A., Ayling, R. and Nicholas, R. 2006. The detection of Mycoplasma (formerly Eperythrozoon) wenyonii by $16 \mathrm{~S}$ rDNA PCR and denaturing gradient gel electrophoresis. Vet. Microbiol. 117: 292-296.

5. Messick, J. B. 2004. Hemotrophic mycoplasmas (hemoplasmas): a review and new insights into pathogenic potential. Vet. Clin. Pathol. 33: 2-13.
6. Neimark, H., Johansson, K. E., Rikihisa, Y. and Tully, J. G. 2001. Proposal to transfer some members of the genera Haemobartonella and Eperythrozoon to the genus Mycoplasma with the descriptions of 'Candidatus Mycoplasma haemofelis', 'Candidatus Mycoplasma haemomuris', 'Candidatus Mycoplasma haemosuis' and 'Candidatus Mycoplasma wenyonii'. Int. J. Syst. Evol. Microbiol. 51: 891-899.

7. Nishizawa, I., Sato, M., Fujihara, M., Sato, S. and Harasawa, R. 2010. Differential detection of hemotropic Mycoplasma species in cattle by melting curve analysis of PCR products. J. Vet. Med. Sci. 72: 77-79. Erratum: J. Vet. Med. Sci. 72: 1704.

8. Su, Q. L., Song, H. Q., Lin, R. Q., Yuan, Z. G., Yang, J. F., Zhao, G. H., Huang, W. Y. and Zhu, X. Q. 2010. The detection of 'Candidatus Mycoplasma haemobos' in cattle and buffalo in China. Trop. Anim. Health Prod. 42: 1805-1808.

9. Tagawa, M., Matsumoto, K. and Inokuma, H. 2008. Molecular detection of Mycoplasma wenyonii and 'Candidatus Mycoplasma haemobos' in cattle in Hokkaido, Japan. Vet. Microbiol. 132: 177-180.

10. Willi, B., Boretti, F. S., Meli, M. L., Bernasconi, M. V., Casati, S., Hegglin, D., Puorger, M., Neimark, H., Cattori, V., Wengi. N., Reusch, C. E., Lutz, H. and Hofmann-Lehmann, R. 2007. Real-time PCR investigation of potential vectors, reservoirs and shedding patterns of feline hemotropic mycoplasmas. Appl. Environ. Microbiol. 73: 3798-3802. 